\title{
Coordination of directional overcurrent and distance relays based on nonlinear multivariable optimization
}

\author{
Tahseen Ali Abd Almuhsen ${ }^{1}$, Ahmed Jasim Sultan² \\ ${ }^{1,2}$ Electrical Engineering Technical Collage, Middle Technical University, Iraq \\ ${ }^{1,2}$ Department of Electrical Power Engineering Techninques, Middle Technical University, Iraq
}

\begin{tabular}{l} 
Article Info \\
\hline Article history: \\
Received Apr 7, 2019 \\
Revised Jul 28, 2019 \\
Accepted Sep 4, 2019 \\
\hline Keywords: \\
Directional overcurrent relay \\
Distane relay \\
Nonlinear multivariable \\
optimization \\
Pilot protection \\
PUTT
\end{tabular}

\begin{abstract}
To ensure stability, security, and protection of electrical equipment from the damage the suitable coordination must be made in interconnected networks. In this paper, the nonlinear multivariable optimization techniques have been used with different performance indexes: Sequential quadratic programming (SQP), Sequential quadratic programming legacy (SQP-Legacy), Interior-Point and Active-Set for IEEE- 8 bus test system. This system consists of twenty-eight protective relays divided into fourteen directional overcurrent relays (DOCR) and fourteen distance relays (DR). It has been tested in the ETAP environment to obtain three-phase short circuit current at the near and far end faults and operating time for all DOC relays for near-end fault as well as test the second zone time for distance relays (TZ2) with pilot signal (WP)and without pilot signal (WOP) of the proposed algorithm was used to reduce overall operating time of DOC relays and obtain optimal values for time multiplier setting (TMS) and TZ2 with the different coordination time interval (CTI) between main and backup relays. The simulation results were validated in ETAP program prove that the effectiveness of the Active-Set to minimize the TMS and TZ2 for the system.
\end{abstract}

Copyright (C) 2020 Institute of Advanced Engineering and Science. All rights reserved.

\section{Corresponding Author:}

Tahseen Ali Abd Almuhsen,

Department of Electrical Power Engineering Techninques,

Middle Technical University,

Baghdad, Iraq.

Email: tahseenengineer@gmail.com

\section{INTRODUCTION}

Protective devices are the watchful eye on the protection of electrical equipment in case of any sudden fault occurred whereby isolated as fast as possible. Commonly distance protection relays are applied as the main protection in high and extra high voltage systems. While directional overcurrent relays are applied as the main protection in medium and low voltage systems and as a backup in high and extra high voltage systems.

Protection relays should be capable to isolate any fault in the network as early as possible so that to reach these goals, coordination between protection relays should be executed [1]. When taking into consideration main and backup relays together are distance protection relays, should be calculated as impedance for three zones as well as take all thing considered of interconnected grids such as the generators and transmission lines in service or out of service [2,3]. There are three sets of coordination problems between DR and DOCR should be determined. These parameter sets are starting current setting $\left(\mathrm{I}_{\text {set }}\right)$, time setting multiple (TSM set) in DOCR and timer of the second zone (TZ2 set) of DR [4, 5].

To get a reliability in the power system should have a suitable setting to each relay, so protection relays should have respective specifications such as speed, selectivity and the sensitivity [6-9]. Conventionally, the protection devices engineers spend more time carry out calculation and employ graphics to coordinate between protection relays with technical constraints. The problem is appearing more difficult with large interrelated transmission grids [10]. 
Presently, the optimization techniques have been used to coordinate between main and backup DOCR as well as between DR and DOCR. Also, it can be resolve constraints after identifying them between main and backup protective relays [10-16]. However, pilot protection has been used to decrease the tripping time of a transmission line [17]. Therefore, the total tripping time reduce from $0.4 \mathrm{sec}$ to $0.04 \mathrm{sec}$, due to communication signal which sends between distance relays that be placed on the same transmission line from both sides [18].

In this paper, a nonlinear multivariable optimization technique with four performance indexes (SQP, SQP-legacy, interior-point, and active-set) for IEEE eight bus system was used to obtain optimal value of (TMS) of DOCR and (TZ2) of DR with and without pilot protection.

\section{PROBLEM FORMULATION}

Any transmission line in power system contains a distance relay as the main protection and directional overcurrent relay as local backup protection as shown in Figure 1. There are three scenarios can be done to achieve coordination between protective relays: main DR with backup DR, main DOCR with backup DOCR, and DOC relay with DR.

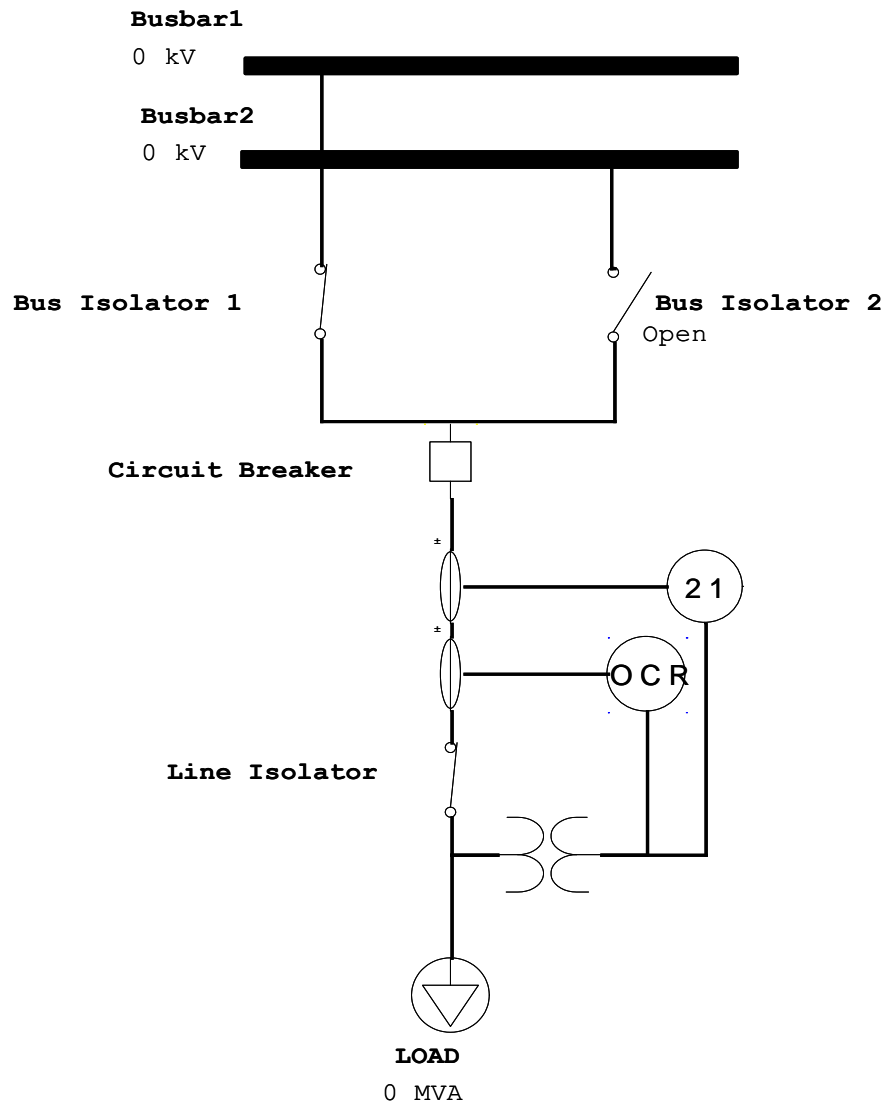

Figure 1. DR with DOCR

Figure 2 shows coordination between main R1 and backup R2 DOCR protective relays for near-end and far-end fault with the following constraints:

$$
\begin{aligned}
& \mathrm{TR} 2(\mathrm{~F} 1)-\mathrm{TR} 1(\mathrm{~F} 1) \geq \mathrm{CTI} 1 \\
& \mathrm{TR} 2(\mathrm{~F} 2)-\mathrm{TR} 1(\mathrm{~F} 2) \geq \mathrm{CTI} 1
\end{aligned}
$$

Where:

TR1(F1) - operating time of main DOCR in near-end fault.

TR1(F1) - operating time of main DOC in far-end fault.

TR2(F1) - operating time of backup DOCR in near-end fault.

Coordination of directional overcurrent and distance relay based on ... (Tahseen Ali Abd Almuhsen) 
TR2(F2) - operating time of backup DOCR in far-end fault.

CTI1 - coordination time interval between $\mathrm{R} 1$ and $\mathrm{R} 2$ relays.

Figure 3 shows coordination between main R3 DR with backup R2 DOCR as well as coordination between main R1 DOCR with backup R4 DR with the following constraints:

$$
\begin{aligned}
& \mathrm{TR} 4(\mathrm{~F} 3)-\mathrm{TR} 1(\mathrm{~F} 3) \geq \mathrm{CTI} 2 \\
& \mathrm{TR} 2(\mathrm{~F} 4)-\mathrm{TR} 3(\mathrm{~F} 4) \geq \mathrm{CTI} 2
\end{aligned}
$$

Where:

TR3(F4) - operating time for second zone of main DR at far-end fault.

TR4(F3) - operating time for second zone of backup DR at near-end fault.

$\mathrm{CTI} 2$ - coordination time interval between $\mathrm{R} 4$ and $\mathrm{R} 1$ relays as well as $\mathrm{R} 2$ and $\mathrm{R} 3$ relays.

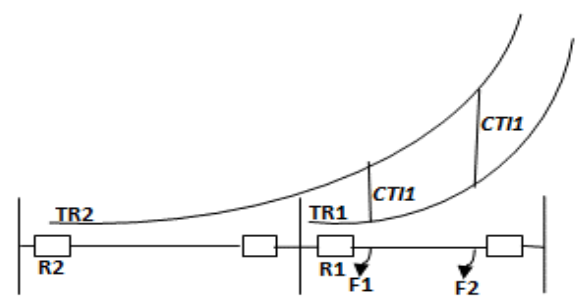

Figure 2. Coordination between main and backup DOCR

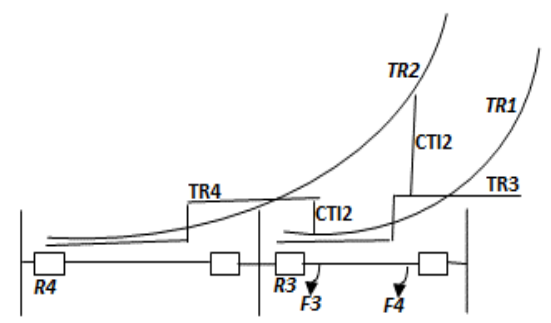

Figure 3. Coordination between DR and DOCRs.

\subsection{Fitness Function for DR and Main DOCR}

The fitness function formula as:

$$
M I N F F=\sum_{j=1}^{M} T_{j}+\sum_{i=1}^{N} T Z 2_{i}
$$

Where:

$\mathrm{FF}$ - fitness function.

$\mathrm{Tj}$ - operating time for $\mathrm{j}^{\text {th }}$ DOC relays for near-end fault.

$\mathrm{M}$ - total number of DOC relays.

TZ2 $\mathrm{i}$ - operating time for second zone $\mathrm{i}^{\mathrm{th}}$ distance relays.

$\mathrm{N}$ - total number of Distance Relays.

\subsection{TMS and Pickup Current Setting of DOCR}

Time multiplier setting (TSM) is bounded between two value lower and upper bound to each relay, as well as pickup current setting $\left(\mathrm{I}_{\mathrm{ps}}\right)$ to each one depends on lower minimum fault current and max load current.

$$
\mathrm{TMS}_{\mathrm{j}} \operatorname{Min} \leq \mathrm{TMS}_{\mathrm{j}} \leq \mathrm{TMS}_{\mathrm{j}} \mathrm{Max}
$$

Where:

$\mathrm{TMS}_{\mathrm{j}}$ Min is minimum bound of TMS for $\mathrm{j}^{\text {th }}$ relays.

$\mathrm{TMS}_{\mathrm{j}}$ max is maximum bound of TMS for $\mathrm{j}^{\text {th }}$ relays.

$$
\mathrm{IPS}_{\mathrm{j}} \text { Max-load } \leq \mathrm{IPS}_{\mathrm{j}} \leq \mathrm{IPS}_{\mathrm{j}} \text { Min-fault }
$$

Where:

IPS $_{\mathrm{j}}$ Max-load - pickup current setting for max load.

IPS $_{\mathrm{j}}$ Min-fault - pickup current setting for min fault. 
According to the bounded value for TMS in equation (6) will be obtain the operating time in equation (1) and (2).in this study according to IEC standard, normal inverse characteristic curve have been used with the following equation [4]:

$$
\mathrm{T}=\left[\frac{0.14}{\left(\frac{I s c}{I p s}\right)^{0.02}-1}\right] \mathrm{TMS}
$$

T - operating time for each DOCR.

$\mathrm{I}_{\mathrm{sc}}$ - secondary value for short circuit current, passing during relay coil.

$\mathrm{I}_{\mathrm{ps}}$ - pickup current setting for each DOC relay.

\section{PILOT PROTECTION}

The role of pilot protection is to accelerate the tripping time between two DR relays at same line and that leads to decrease the total operating time as well as operating time for second zone of DR. The permissive under reach transfer trip (PUTT) signal used to accelerate the tripping time [19]. The PUTT philosophy can be shown in Figure 4.

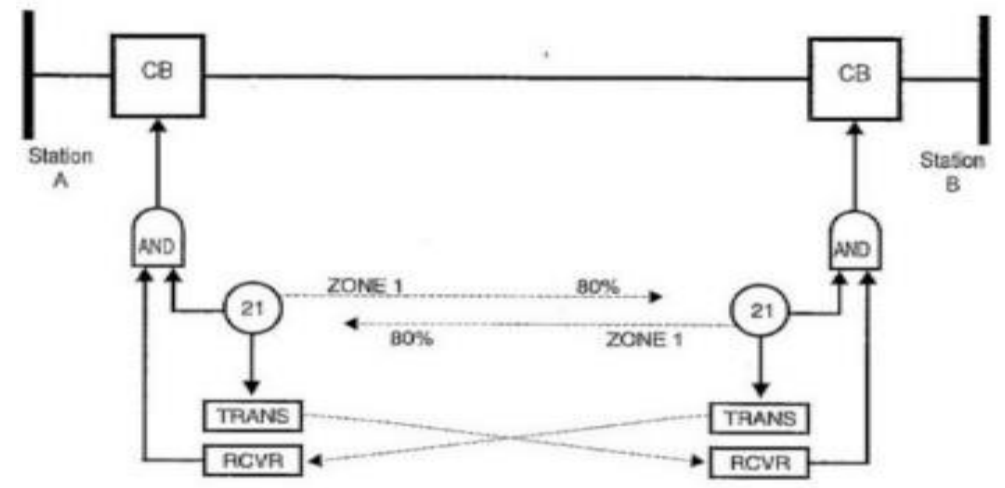

Figure 4. PUTT philosophy

The transmission line would be divided into three zons: first zone have $80 \%$ of protected line length with instantaneous operating time, second zone has imp but the second zone setting $120 \%$ of line length impedance at operating time equal to 0.4 , so use PUTT to accelerate trip when one of DR see the fault at the second zone $(80 \%-100 \%)$ will start the second zone and receive signal from the remote distance relay and collect in (AND GATE), send trip to local circuit breaker and Reduces trip time from 40 to 2-4 millisecond. Figure 3 will be as shown in Figure 5 the second zone for the main distance relay time will reduce to 0.04 second.

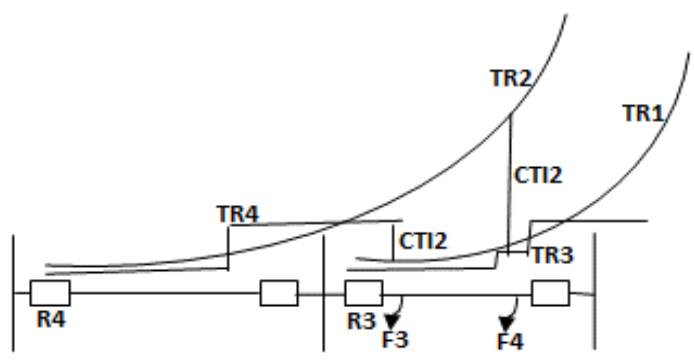

Figure 5. Coordination between distance and DOC relays with pilot protection 


\section{NONLINEAR MULTIVARIABLE ALGORITHM}

Figure 6 shows the flow chart for the nonlinear multivariable optimization for solve coordination problem between main and back up relays.

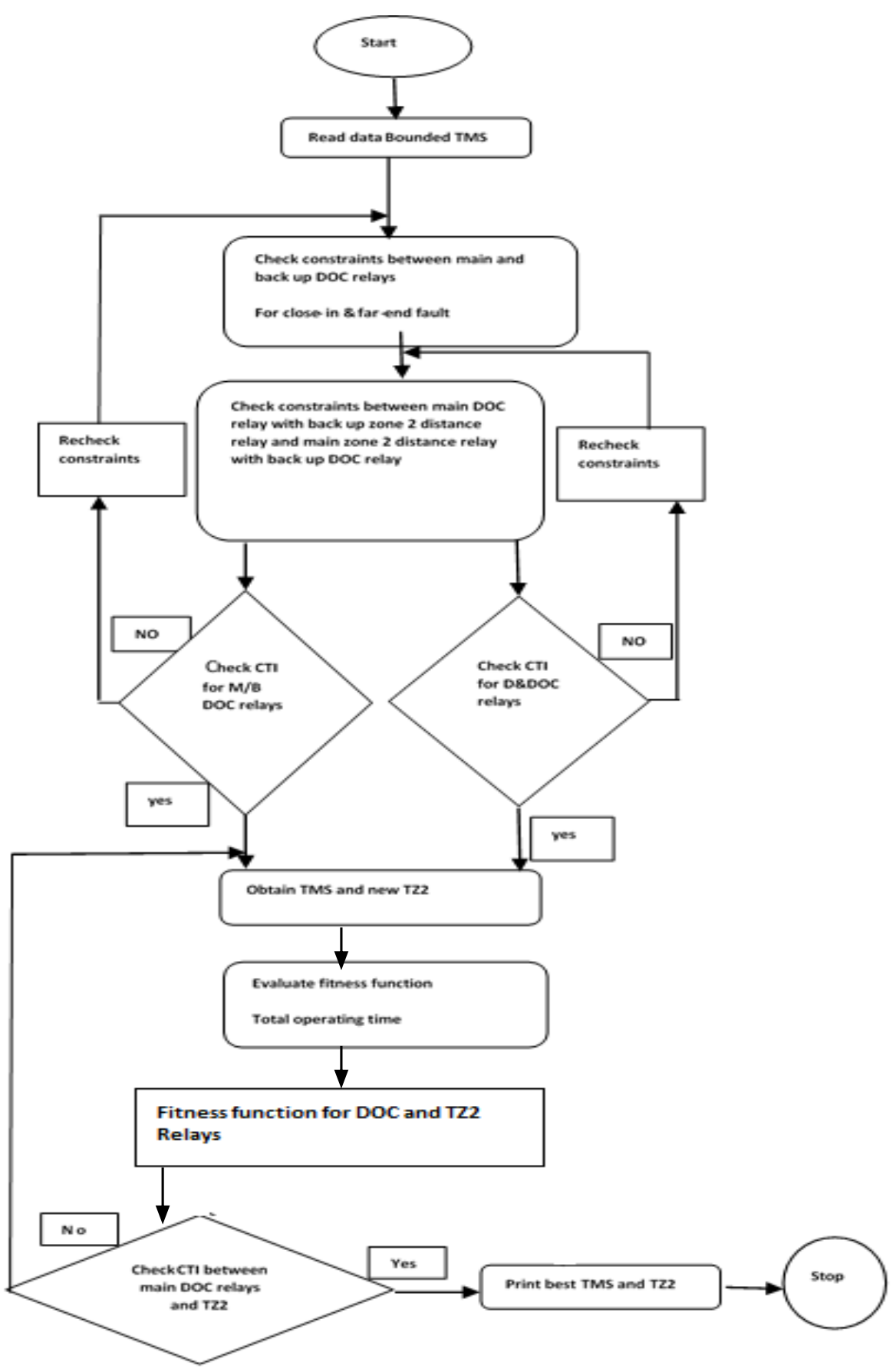

Figure 6. Flowchart for proposed algorithm

\section{RESULTS AND DISCUSSION}

The IEEE- eight bus system consists of seven transmission lines, two generators, two step-up transformers and extension network at bus four with 400 MVA short circuit [20]. Therefore, we have fourteen distance relays and fourteen DOC relays according to the number of transmission lines. The pick-up current setting and current transformer ratio data presented in Table 1. 
Table 1. Pick Up Current and Current Transformer Ratio

\begin{tabular}{cccccc}
\hline No of relay & Pick up setting(A) & Current transformer ratio & No of relay & Pick up setting(A) & Current transformer ratio \\
\hline R1 & 1 & 240 & R8 & 2.5 & 2 \\
R2 & 2.5 & 240 & R9 & 2.5 & 160 \\
R3 & 2.5 & 160 & R10 & 2.5 & 240 \\
R4 & 2.5 & 240 & R11 & 2.5 & 240 \\
R5 & 1.5 & 240 & R12 & 1.5 & 240 \\
R6 & 2.5 & 240 & R13 & 0.5 & 240 \\
R7 & 0.5 & 160 & R14 & & 160 \\
\hline
\end{tabular}

In Table 2 used the ETAP program to obtain three-phase short circuit current for near and far-end faults and Figure 7 shows the test system.

Table 2. Three Phase Short Circuit Current for Near and Far End Faults

\begin{tabular}{|c|c|c|c|c|c|c|c|}
\hline \multicolumn{4}{|c|}{ Three phase close-in end fault } & \multicolumn{4}{|c|}{ Three phase far end fault } \\
\hline $\begin{array}{c}\text { Primary } \\
\text { Relay }\end{array}$ & $\begin{array}{l}\text { Fault current } \\
\text { (A) }\end{array}$ & $\begin{array}{c}\text { Back upRelay } \\
\text { (A) }\end{array}$ & $\begin{array}{c}\text { Fault current } \\
\text { (A) }\end{array}$ & Primary Relay & $\begin{array}{c}\text { Fault } \\
\text { current }(\mathrm{A})\end{array}$ & $\begin{array}{c}\text { Back up } \\
\text { Relay (A) }\end{array}$ & $\begin{array}{c}\text { Fault current } \\
\text { (A) }\end{array}$ \\
\hline $\mathrm{R} 1$ & 3069 & R6 & 3069 & R1 & 935 & R6 & 935 \\
\hline $\mathrm{R} 2$ & 5459 & R1 & 935 & $\mathrm{R} 2$ & 3364 & $\mathrm{R} 1$ & 380 \\
\hline $\mathrm{R} 2$ & 5459 & $\mathrm{R} 7$ & 1775 & $\mathrm{R} 2$ & 3364 & $\mathrm{R} 7$ & 721 \\
\hline $\mathrm{R} 3$ & 3364 & $\mathrm{R} 2$ & 3364 & $\mathrm{R} 3$ & 2120 & $\mathrm{R} 2$ & 2120 \\
\hline $\mathrm{R} 4$ & 3659 & $\mathrm{R} 3$ & 2120 & $\mathrm{R} 4$ & 2337 & $\mathrm{R} 3$ & 969 \\
\hline R5 & 2337 & $\mathrm{R} 4$ & 2337 & R5 & 1176 & $\mathrm{R} 4$ & 1176 \\
\hline R6 & 5682 & R5 & 1176 & R6 & 3069 & $\mathrm{R} 5$ & 646 \\
\hline R6 & 5682 & R14 & 1758 & R6 & 3069 & R14 & $74 *$ \\
\hline R7 & 4851 & R5 & 1176 & R7 & 1775 & R5 & $221 *$ \\
\hline R7 & 4851 & R13 & 927 & R7 & 1775 & $\mathrm{R} 13$ & $935 \#$ \\
\hline R8 & 5667 & R7 & 1775 & $\mathrm{R} 8$ & 2838 & R7 & 74\# \\
\hline R8 & 5667 & R9 & 1144 & $\mathrm{R} 8$ & 2838 & R9 & 575 \\
\hline R9 & 2418 & R10 & 2418 & R9 & 1144 & R10 & 1144 \\
\hline R10 & 3756 & R11 & 2217 & R10 & 2418 & R11 & 1056 \\
\hline R11 & 3501 & R12 & 3501 & R11 & 2217 & $\mathrm{R} 12$ & 2217 \\
\hline $\mathrm{R} 12$ & 5434 & R13 & 927 & R12 & 3501 & R13 & 417 \\
\hline R12 & 5434 & R14 & 1758 & R12 & 3501 & R14 & 792 \\
\hline R13 & 2838 & $\mathrm{R} 8$ & 2838 & R13 & 927 & $\mathrm{R} 8$ & 927 \\
\hline R14 & 4828 & $\mathrm{R} 1$ & 935 & R14 & 1758 & $\mathrm{R} 1$ & 927\# \\
\hline R14 & 4828 & R9 & 1144 & R14 & 1758 & R9 & $192^{*}$ \\
\hline
\end{tabular}

Symbol * shown the current which not reach to pick up current setting during passing in DOC relays.

Symbol \# shows the current which passing during DOC relays but in reverse directional.

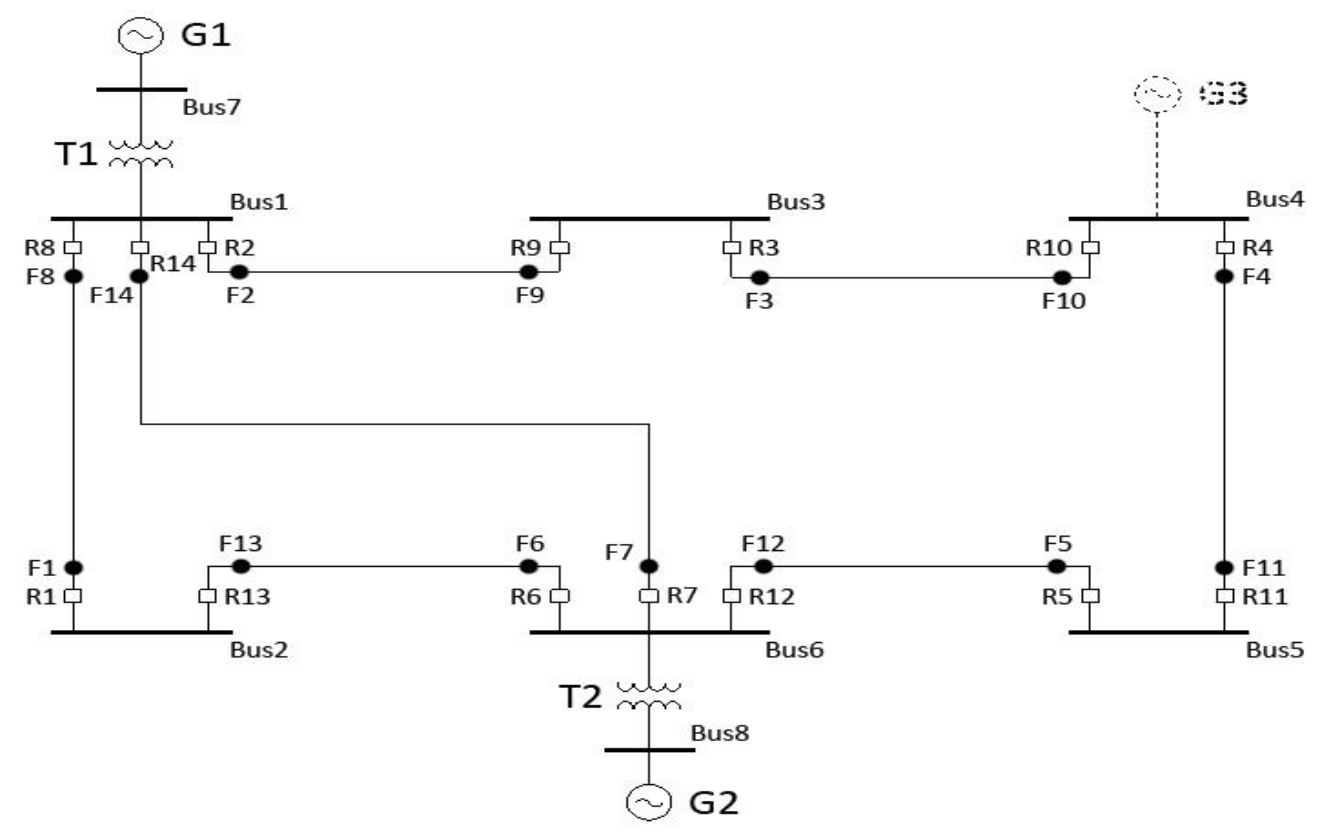

Figure 7. The test system 
In [20-25] the range of coordination time interval is $(0.2-0.5)$ second. So the CTI1 and CTI2 in equation from 1 to 4 will be chosen equal to 0.2 seconds in case (1) and in case (2) CTI $=0.3$ in equation 1 and 2 and CTI $2=0.2$ in equation 3 and 4 and the TMS has been bounded from 0.1 to 1.1 continuous for lower and upper bounds respectively to each relay. Choosing the zones timer setting for each distance relay is $\mathrm{TZ} 1=0, \mathrm{TZ2}=0.4$, and $\mathrm{TZ3}=0.8$.

The test for the system has been done with four performance indexes for nonlinear multivariable optimization technique is sequential quadratic programming (SQP), sequential quadratic programming legacy (SQP-legacy), interior - point and active - set with pilot protection(WP) and without pilot protection (WOP). There are sixty-eight linear inequality constraints and twenty- eight variable, all these constraints during the test in MATLAB simulation have achieved. Table 3 shows the number of iterations and elapsed time to find the solution for the four algorithms which used with and without pilot protection for two cases $1 \& 2$.

Table 3. The Nonlinear Multivariable Optimization with Different Performance Indexes for All Cases

\begin{tabular}{|c|c|c|c|c|c|c|c|c|}
\hline \multirow{3}{*}{ Algorithms } & \multicolumn{4}{|c|}{ Case(1) } & \multicolumn{4}{|c|}{ Case (2) } \\
\hline & \multicolumn{2}{|c|}{ No of iteration } & \multicolumn{2}{|c|}{ The solving time in (sec) } & \multicolumn{2}{|c|}{ No of iteration } & \multicolumn{2}{|c|}{ The solving time in $(\mathrm{sec})$} \\
\hline & WP & WOP & WP & WOP & WP & WOP & WP & WOP \\
\hline SQP & 3 & 3 & 2.6684 & 2.2103 & 3 & 3 & 2.3007 & 2.2707 \\
\hline SQP-legacy & 3 & 3 & 2.3874 & 2.3574 & 3 & 3 & 2.3285 & 2.5367 \\
\hline Interior-point & 13 & 13 & 4.2236 & 3.5995 & 14 & 15 & 4.2596 & 4.1416 \\
\hline Active- set & 2 & 2 & 2.1552 & 0.7059 & 2 & 2 & 2.2788 & 2.2292 \\
\hline
\end{tabular}

According to results, the active-set performance index was the least time and least iterations to obtain optimal value of TMS for all DOC relays and TZ2 for all distance relays with and without pilot signal protection in all cases.

All algorithms with and without pilot signal the results of TMS for DOC relays from relay 1 to relay 14 and the second zone timer for distance relays from relay 15 to relay 28 as identical results for all cases. all these results for case 1 and 2 show in Table 4 and Table 5 with total minimum fitness function of near-end faults for DOC relays as well as the fitness function for distance relays at second zone respectively. The overall time for DOC relays reduced about 3.18 MS in case 1 and 4.77 MS in case 2 when tested with and without pilot protection.

Table 4. TMS for DOC Relays with All Performance Indexes

\begin{tabular}{|c|c|c|c|c|c|c|c|}
\hline \multicolumn{4}{|c|}{$\begin{array}{c}\text { TMS for all performance indexes } \\
\text { (sqp, sqp-legacy, active-set and interior point). } \\
\text { At CTI }=0.2 \text { between (main and backup DOC relays) } \\
\text { CASE (1) }\end{array}$} & \multicolumn{4}{|c|}{$\begin{array}{c}\text { TMS for all performance indexes } \\
\text { (sqp, sqp-legacy, active-set and interior point). } \\
\text { At CTI }=0.3 \text { between (main and backup DOC relays) } \\
\text { CASE (2) }\end{array}$} \\
\hline $\begin{array}{l}\text { No of } \\
\text { Relay }\end{array}$ & With pilot & Without pilot & $\begin{array}{l}\text { Only with near } \\
\text { end fault }\end{array}$ & $\begin{array}{l}\text { No of } \\
\text { Relay }\end{array}$ & With pilot & $\begin{array}{c}\text { Without } \\
\text { pilot }\end{array}$ & $\begin{array}{c}\text { Only with near } \\
\text { end fault }\end{array}$ \\
\hline R1 & 0.1562 & 0.1562 & 0.1562 & $\mathrm{R} 1$ & 0.2343 & 0.2343 & 0.2343 \\
\hline $\mathrm{R} 2$ & 0.1913 & 0.1913 & 0.1913 & $\mathrm{R} 2$ & 0.287 & 0.287 & 0.287 \\
\hline R3 & 0.1751 & 0.1751 & 0.1751 & R3 & 0.2627 & 0.2627 & 0.2627 \\
\hline $\mathrm{R} 4$ & 0.1375 & 0.1375 & 0.1375 & $\mathrm{R} 4$ & 0.2063 & 0.2063 & 0.2063 \\
\hline R5 & 0.1357 & 0.1357 & 0.1357 & R5 & 0.2035 & 0.2035 & 0.2035 \\
\hline R6 & 0.1465 & 0.1465 & 0.1465 & R6 & 0.2198 & 0.2198 & 0.2198 \\
\hline R7 & 0.3623 & 0.3623 & 0.3623 & R7 & 0.5435 & 0.5435 & 0.5435 \\
\hline $\mathrm{R} 8$ & 0.1261 & 0.1261 & 0.1261 & $\mathrm{R} 8$ & 0.1891 & 0.1891 & 0.1891 \\
\hline R9 & 0.1447 & 0.1447 & 0.1447 & R9 & 0.217 & 0.217 & 0.217 \\
\hline $\mathrm{R} 10$ & 0.131 & 0.131 & 0.1395 & $\mathrm{R} 10$ & 0.1965 & 0.1965 & 0.2092 \\
\hline $\mathrm{R} 11$ & 0.1367 & 0.1367 & 0.1367 & $\mathrm{R} 11$ & 0.205 & 0.205 & 0.205 \\
\hline $\mathrm{R} 12$ & 0.188 & 0.188 & 0.188 & $\mathrm{R} 12$ & 0.282 & 0.282 & 0.282 \\
\hline $\mathrm{R} 13$ & 0.1081 & 0.1081 & 0.1081 & $\mathrm{R} 13$ & 0.1622 & 0.1622 & 0.1622 \\
\hline $\mathrm{R} 14$ & $0 . .3570$ & $0 . .3570$ & $0 . .3570$ & R14 & 0.5355 & 0.5355 & 0.5355 \\
\hline$\sum_{j=1}^{M} T_{j}$ & 7.0615 & 7.0615 & 7.0933 & $\sum_{j=1}^{M} T j$ & 10.5923 & 10.5923 & 10.640 \\
\hline
\end{tabular}


Table 5. TZ2 for Distance Relays with All Performance Indexes

\begin{tabular}{|c|c|c|c|c|c|c|c|}
\hline \multicolumn{4}{|c|}{$\begin{array}{c}\text { TZ2 for all performance indexes } \\
\text { (Sqp, sqp-legacy, active-set and interior point). } \\
\text { At CTI = 0.2 between (main and backup DOC relays) } \\
\text { CASE (1) }\end{array}$} & \multicolumn{4}{|c|}{$\begin{array}{c}\text { TZ2 for all performance indexes } \\
\text { (Sqp, sqp-legacy, active-set and interior point). } \\
\text { At CTI }=0.2 \text { between (main and backup DOC relays) } \\
\text { CASE (2) }\end{array}$} \\
\hline $\begin{array}{l}\text { No of } \\
\text { Relay }\end{array}$ & With pilot & Without pilot & $\begin{array}{l}\text { Without using } \\
\text { far end fault, } \\
\text { with and } \\
\text { without pilot. }\end{array}$ & $\begin{array}{l}\text { No of } \\
\text { Relay }\end{array}$ & With pilot & Without pilot & $\begin{array}{l}\text { Without using } \\
\text { far end fault, } \\
\text { with and } \\
\text { without pilot. }\end{array}$ \\
\hline R15 & 0.7932 & 0.7932 & 0.7932 & R15 & 1.0898 & 1.0898 & 1.0898 \\
\hline $\mathrm{R} 16$ & 0.9228 & 0.9228 & 0.9228 & $\mathrm{R} 16$ & 1.2842 & 1.2842 & 1.2842 \\
\hline R17 & 0.7228 & 0.7228 & 0.7228 & $\mathrm{R} 17$ & 0.9842 & 0.9842 & 0.9842 \\
\hline $\mathrm{R} 18$ & 0.6983 & 0.6983 & 0.6983 & $\mathrm{R} 18$ & 0.9474 & 0.9474 & 0.9474 \\
\hline R19 & 0.7928 & 0.7928 & 0.7928 & R19 & 1.0893 & 1.0893 & 1.0893 \\
\hline $\mathrm{R} 20$ & 0.6182 & 0.6182 & 0.6182 & $\mathrm{R} 20$ & 0.8273 & 0.8273 & 0.8273 \\
\hline $\mathrm{R} 21$ & 0.7932 & 0.7932 & 0.7932 & $\mathrm{R} 21$ & 1.0898 & 1.0898 & 1.0898 \\
\hline $\mathrm{R} 22$ & 0.5591 & 0.5591 & 0.5591 & $\mathrm{R} 22$ & 0.7387 & 0.7387 & 0.7387 \\
\hline $\mathrm{R} 23$ & 0.7849 & 0.7849 & 0.7849 & $\mathrm{R} 23$ & 1.0773 & 1.0773 & 1.0773 \\
\hline $\mathrm{R} 24$ & 0.6907 & 0.6907 & 0.6907 & $\mathrm{R} 24$ & 0.9361 & 0.9361 & 0.9361 \\
\hline $\mathrm{R} 25$ & 0.7225 & 0.7225 & 0.7225 & $\mathrm{R} 25$ & 0.9838 & 0.9838 & 0.9838 \\
\hline $\mathrm{R} 26$ & 0.7329 & 0.7329 & 0.7329 & $\mathrm{R} 26$ & 0.9994 & 0.9994 & 0.9994 \\
\hline $\mathrm{R} 27$ & 0.7928 & 0.7928 & 0.7928 & $\mathrm{R} 27$ & 1.0893 & 1.0893 & 1.0893 \\
\hline R28 & 0.7841 & 0.7841 & 0.7841 & R28 & 1.0762 & 1.0762 & 1.0762 \\
\hline$\sum_{i=1}^{N} T Z 2 i$ & 10.4084 & 10.4084 & 10.4084 & $\sum_{i=1}^{N} T Z 2 i$ & 14.2126 & 14.2126 & 14.2126 \\
\hline $\begin{array}{c}\text { Average } \\
\text { TZ2 }\end{array}$ & 0.7435 & 0.7435 & 0.7435 & $\begin{array}{c}\text { Average } \\
\text { TZ2 }\end{array}$ & 1.0152 & 1.0152 & 1.0152 \\
\hline
\end{tabular}

The operating time for DOC relays in Matlab and ETAP simulation as well as the second zone timing and the timing of third zone will be $($ TZ2 + 0.4) shown in Table 6 and represent as a bar chart in Figure 8 for case 1 and Table 7 and Figure 9 for case (2).

Table 6. The Operating Time for DOC Relays and Distance Relays (Case1)

\begin{tabular}{|c|c|c|c|c|c|c|c|c|c|c|}
\hline $\begin{array}{l}\text { NO of } \\
\text { Main } \\
\text { relay }\end{array}$ & $\begin{array}{c}\text { Time } \\
\text { main } \\
\text { DOCR } \\
\text { in } \\
\text { matlab } \\
(\mathrm{sec})\end{array}$ & $\begin{array}{c}\text { Time } \\
\text { main } \\
\text { DOCR } \\
\text { in ETAP } \\
(\mathrm{sec})\end{array}$ & $\begin{array}{c}\text { NO of } \\
\text { backup } \\
\text { relay }\end{array}$ & $\begin{array}{c}\text { Time } \\
\text { backup } \\
\text { DOCR in } \\
\text { matlab } \\
(\mathrm{sec})\end{array}$ & $\begin{array}{c}\text { Time } \\
\text { main } \\
\text { DOCR } \\
\text { in } \\
\text { ETAP } \\
(\mathrm{sec})\end{array}$ & $\begin{array}{l}\text { NO of } \\
\text { relay }\end{array}$ & $\begin{array}{c}\text { TZ2 } \\
\text { distance } \\
\text { Relay }(\mathrm{sec})\end{array}$ & $\begin{array}{c}\text { TZ3 } \\
\text { distance } \\
\text { relay }(\mathrm{sec})\end{array}$ & $\begin{array}{c}\text { CTI } \\
\text { between } \\
\text { Main and } \\
\text { backup } \\
\text { DOC } \\
\text { relay }\end{array}$ & $\begin{array}{c}\text { CTI } \\
\text { between } \\
\text { Main } \\
\text { DOC } \\
\text { relay and } \\
\text { distance } \\
\text { relay }\end{array}$ \\
\hline $\mathrm{R} 1$ & 0.4182 & 0.418 & R6 & 0.6182 & 0.616 & R20 & 0.6182 & 1.0182 & 0.2 & 0.2 \\
\hline \multirow[b]{2}{*}{$\mathrm{R} 2$} & \multirow{2}{*}{0.5932} & \multirow{2}{*}{0.592} & $\mathrm{R} 1$ & 0.7932 & 0.792 & $\mathrm{R} 15$ & 0.7932 & 1.1932 & 0.2 & 0.2 \\
\hline & & & R7 & 0.7932 & 0.792 & $\mathrm{R} 21$ & 0.7932 & 1.1932 & 0.2 & 0.2 \\
\hline R3 & 0.5634 & 0.563 & $\mathrm{R} 2$ & 0.7634 & 0.762 & R16 & 0.9228 & 1.3228 & 0.2 & 0.3594 \\
\hline $\mathrm{R} 4$ & 0.5228 & 0.525 & R3 & 0.7228 & 0.722 & $\mathrm{R} 17$ & 0.7228 & 1.1228 & 0.2 & 0.2 \\
\hline R5 & 0.4983 & 0.500 & $\mathrm{R} 4$ & 0.6983 & 0.701 & $\mathrm{R} 18$ & 0.6983 & 1.0983 & 0.2 & 0.2 \\
\hline \multirow{2}{*}{ R6 } & \multirow{2}{*}{0.4461} & \multirow{2}{*}{0.444} & R5 & 0.7928 & 0.795 & R19 & 0.7928 & 1.1928 & 0.3467 & 0.3467 \\
\hline & & & $\mathrm{R} 14$ & 0.7841 & 0.784 & $\mathrm{R} 28$ & 0.7841 & 1.1841 & 0.338 & 0.338 \\
\hline \multirow{2}{*}{ R7 } & \multirow{2}{*}{0.5928} & \multirow{2}{*}{0.592} & R5 & 0.7928 & 0.795 & R19 & 0.7928 & 1.1928 & 0.2 & 0.2 \\
\hline & & & R13 & 0.7928 & 0.792 & $\mathrm{R} 27$ & 0.7928 & 1.1924 & 0.2 & 0.2 \\
\hline \multirow{2}{*}{$\mathrm{R} 8$} & \multirow{2}{*}{0.3842} & \multirow{2}{*}{0.384} & $\mathrm{R} 7$ & 0.7932 & 0.792 & $\mathrm{R} 21$ & 0.7932 & 1.1932 & 0.409 & 0.409 \\
\hline & & & R9 & 0.7849 & 0.787 & $\mathrm{R} 23$ & 0.7849 & 1.1849 & 0.4007 & 0.4007 \\
\hline R9 & 0.4907 & 0.492 & $\mathrm{R} 10$ & 0.6486 & 0.649 & $\mathrm{R} 24$ & 0.6907 & 1.0907 & 0.1579 & 0.2 \\
\hline $\mathrm{R} 10$ & 0.4907 & 0.491 & R11 & 0.7225 & 0.724 & $\mathrm{R} 25$ & 0.7225 & 1.1225 & 0.2318 & 0.2318 \\
\hline R11 & 0.5329 & 0.534 & $\mathrm{R} 12$ & 0.7329 & 0.733 & $\mathrm{R} 26$ & 0.7329 & 1.1329 & 0.2 & 0.2 \\
\hline \multirow{2}{*}{$\mathrm{R} 12$} & \multirow{2}{*}{0.5841} & \multirow{2}{*}{0.584} & R13 & 0.7928 & 0.792 & $\mathrm{R} 27$ & 0.7928 & 1.1924 & 0.2087 & 0.2087 \\
\hline & & & R14 & 0.7841 & 0.784 & $\mathrm{R} 28$ & 0.7841 & 1.1841 & 0.2 & 0.2 \\
\hline $\mathrm{R} 13$ & 0.3591 & 0.359 & $\mathrm{R} 8$ & 0.5591 & 0.559 & R22 & 0.5591 & 0.9591 & 0.2 & 0.2 \\
\hline \multirow{2}{*}{$\mathrm{R} 14$} & \multirow{2}{*}{0.5849} & \multirow{2}{*}{0.585} & $\mathrm{R} 1$ & 0.7932 & 0.792 & $\mathrm{R} 15$ & 0.7932 & 1.1932 & 0.2083 & 0.2083 \\
\hline & & & $\mathrm{R} 9$ & 0.7849 & 0.787 & $\mathrm{R} 23$ & 0.7849 & 1.1849 & 0.2 & 0.2 \\
\hline
\end{tabular}


Table 7. The Operating Time for DOC Relays and Distance Relays (Case2)

\begin{tabular}{|c|c|c|c|c|c|c|c|c|c|c|}
\hline $\begin{array}{l}\text { NO of } \\
\text { Main } \\
\text { relay }\end{array}$ & $\begin{array}{c}\text { Time } \\
\text { main } \\
\text { DOCR } \\
\text { in } \\
\text { Matlab } \\
(\mathrm{sec})\end{array}$ & $\begin{array}{c}\text { Time } \\
\text { main } \\
\text { DOCR } \\
\text { in } \\
\text { ETAP } \\
(\mathrm{sec})\end{array}$ & $\begin{array}{l}\text { NO of } \\
\text { backup } \\
\text { relay }\end{array}$ & $\begin{array}{c}\text { Time } \\
\text { backup } \\
\text { DOCR } \\
\text { in } \\
\text { Matlab } \\
(\mathrm{sec})\end{array}$ & $\begin{array}{c}\text { Time } \\
\text { main } \\
\text { DOCR } \\
\text { in } \\
\text { ETAP } \\
(\mathrm{sec})\end{array}$ & $\begin{array}{l}\text { NO } \\
\text { of } \\
\text { relay }\end{array}$ & $\begin{array}{c}\text { TZ2 } \\
\text { distance } \\
\text { Relay }(\mathrm{sec})\end{array}$ & $\begin{array}{c}\text { TZ3 } \\
\text { distance } \\
\text { relay }(\mathrm{sec})\end{array}$ & $\begin{array}{c}\text { CTI } \\
\text { between } \\
\text { Main } \\
\text { and } \\
\text { backup } \\
\text { DOC } \\
\text { relay }\end{array}$ & $\begin{array}{c}\text { CTI } \\
\text { between } \\
\text { Main } \\
\text { DOC } \\
\text { relay } \\
\text { and } \\
\text { distance } \\
\text { relay }\end{array}$ \\
\hline R1 & 0.6273 & 0.627 & R6 & 0.9273 & 0.928 & R20 & 0.8273 & 1.2273 & 0.3 & 0.2 \\
\hline \multirow{2}{*}{$\mathrm{R} 2$} & \multirow{2}{*}{0.8898} & \multirow{2}{*}{0.890} & $\mathrm{R} 1$ & 1.1898 & 1.188 & $\mathrm{R} 15$ & 1.0898 & 1.4898 & 0.3 & 0.2 \\
\hline & & & R7 & 1.1898 & 1.189 & R21 & 1.0898 & 1.4898 & 0.3 & 0.2 \\
\hline R3 & 0.8452 & 0.846 & $\mathrm{R} 2$ & 1.1452 & 1.145 & R16 & 1.2842 & 1.6842 & 0.3 & 0.439 \\
\hline $\mathrm{R} 4$ & 0.7842 & 0.783 & R3 & 1.0842 & 1.085 & R17 & 0.9842 & 1.3842 & 0.3 & 0.2 \\
\hline R5 & 0.7474 & 0.746 & $\mathrm{R} 4$ & 1.0474 & 1.046 & R18 & 0.9474 & 1.3474 & 0.3 & 0.2 \\
\hline \multirow{2}{*}{ R6 } & \multirow{2}{*}{0.6691} & \multirow{2}{*}{0.670} & R5 & 1.1893 & 1.186 & R19 & 1.0893 & 1.4893 & 0.5202 & 0.4202 \\
\hline & & & R14 & 1.1762 & 1.175 & $\mathrm{R} 28$ & 1.0762 & 1.4762 & 0.5071 & 0.4071 \\
\hline \multirow{2}{*}{ R7 } & \multirow{2}{*}{0.8893} & \multirow{2}{*}{0.889} & R5 & 1.1893 & 1.186 & R19 & 1.0893 & 1.4893 & 0.3 & 0.2 \\
\hline & & & R13 & 1.1893 & 1.188 & $\mathrm{R} 27$ & 1.0893 & 1.4893 & 0.3 & 0.2 \\
\hline \multirow{2}{*}{$\mathrm{R} 8$} & \multirow{2}{*}{0.5764} & \multirow{2}{*}{0.576} & $\mathrm{R} 7$ & 1.1898 & 1.189 & $\mathrm{R} 21$ & 1.0898 & 1.4898 & 0.6134 & 0.5134 \\
\hline & & & R9 & 1.1773 & 1.177 & $\mathrm{R} 23$ & 1.0773 & 1.4773 & 0.6009 & 0.5009 \\
\hline R9 & 0.7361 & 0.736 & R10 & 0.973 & 0.976 & $\mathrm{R} 24$ & 0.9361 & 1.3361 & 0.2369 & 0.2 \\
\hline $\mathrm{R} 10$ & 0.7361 & 0.738 & $\mathrm{R} 11$ & 1.0838 & 1.084 & $\mathrm{R} 25$ & 0.9838 & 1.3838 & 0.3477 & 0.2477 \\
\hline $\mathrm{R} 11$ & 0.7994 & 0.799 & $\mathrm{R} 12$ & 1.0994 & 1.099 & $\mathrm{R} 26$ & 0.9994 & 1.3994 & 0.3 & 0.2 \\
\hline \multirow{2}{*}{ R12 } & \multirow{2}{*}{0.8762} & \multirow{2}{*}{0.876} & $\mathrm{R} 13$ & 1.1893 & 1.188 & $\mathrm{R} 27$ & 1.0893 & 1.4893 & 0.3131 & 0.2131 \\
\hline & & & $\mathrm{R} 14$ & 1.1762 & 1.175 & $\mathrm{R} 28$ & 1.0762 & 1.4762 & 0.3 & 0.2 \\
\hline $\mathrm{R} 13$ & 0.5387 & 0.538 & $\mathrm{R} 8$ & 0.8387 & 0.838 & $\mathrm{R} 22$ & 0.7387 & 1.1387 & 0.3 & 0.2 \\
\hline \multirow{2}{*}{ R14 } & \multirow{2}{*}{0.8773} & \multirow{2}{*}{0.877} & $\mathrm{R} 1$ & 1.1898 & 1.188 & $\mathrm{R} 15$ & 1.0898 & 1.4898 & 0.3125 & 0.2125 \\
\hline & & & $\mathrm{R} 9$ & 1.1773 & 1.177 & $\mathrm{R} 23$ & 1.0773 & 1.4773 & 0.3 & 0.2 \\
\hline
\end{tabular}

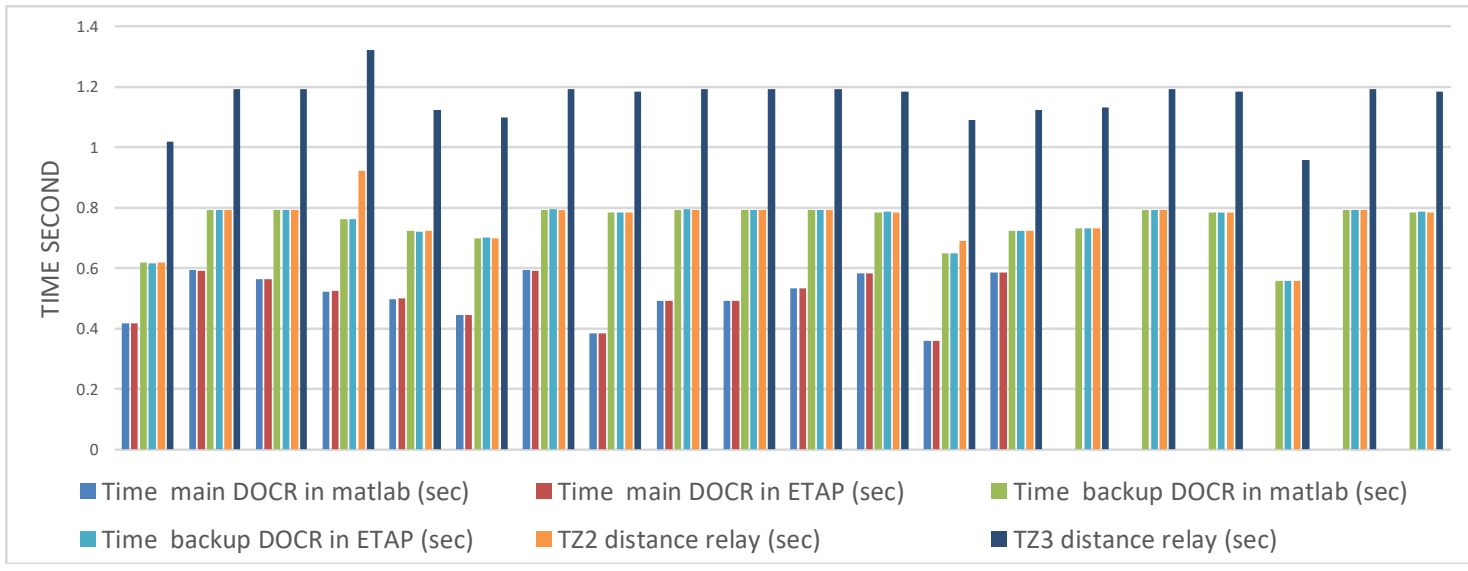

Figure 8. The operating time for DOC relays

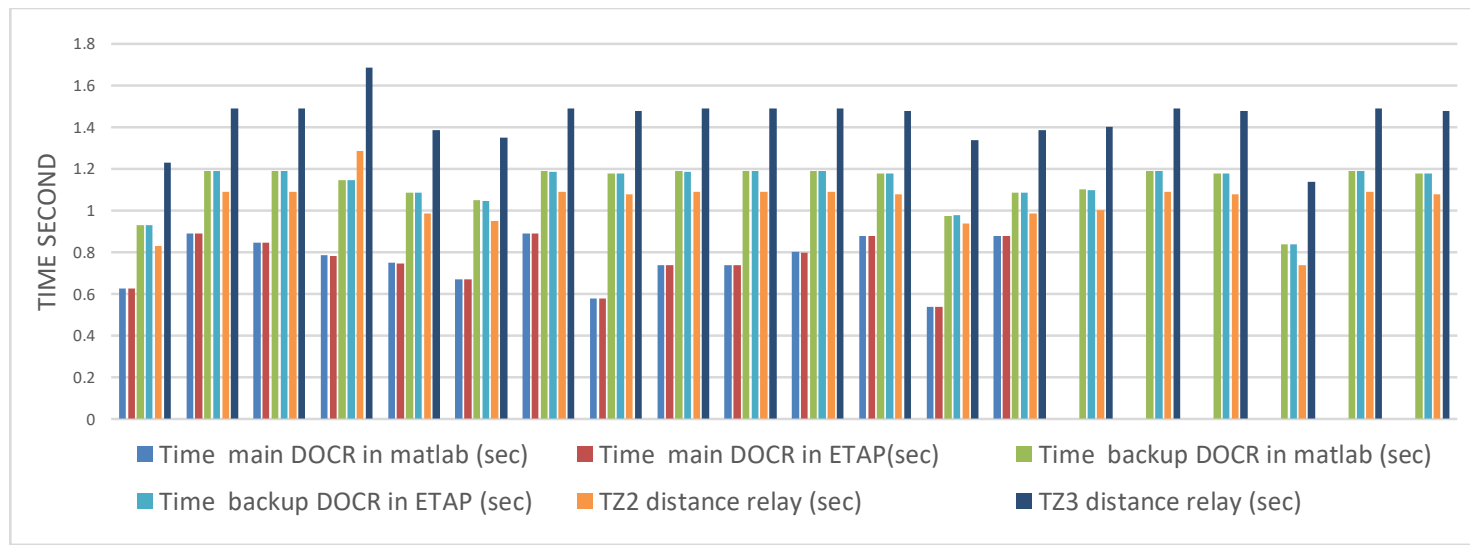

Figure 9. The operating time for DOC relays and distance relays (case1) distance relays (case2) 
The system has been tested in ETAP environment for all DOC relays during near-end fault to obtain the real operating time according to TMS curve for each relay. All relays chose same type Siemens type 7SJ64 numerical relay except R7 and R14 chose ABB relay type REF630, because of the operating time which obtained was wrong with Siemens relay the reason was the pick up current for Siemens type start from 0.5 A secondary current and the pick-up current for R7 and R14 is $0.5 \mathrm{~A}$ that is lead to wrong results during test, while the ABB relay, the pick-up current start from 0.05A secondary current and this type more sensitive with pick up current for the R7 and R14 relays. One of these tests was at the transmission line (1-2) near from R1 for case 1 and 2 and the results of operating time for R1 and back up relays which had been sensitive by fault, shown in Figure 10 and Figure 11 respectively.

In case 1 the operating time for back up DOC relays and the second zone time for distance relays often will be trip in same time during fault, if the main zone 1 of distance relay and main DOC relay will failure and in case 2, if the main zone 1 and main DOC relay fails to clear faults the priority will be for second zone time for distance relay to clear fault before back up DOC relay.

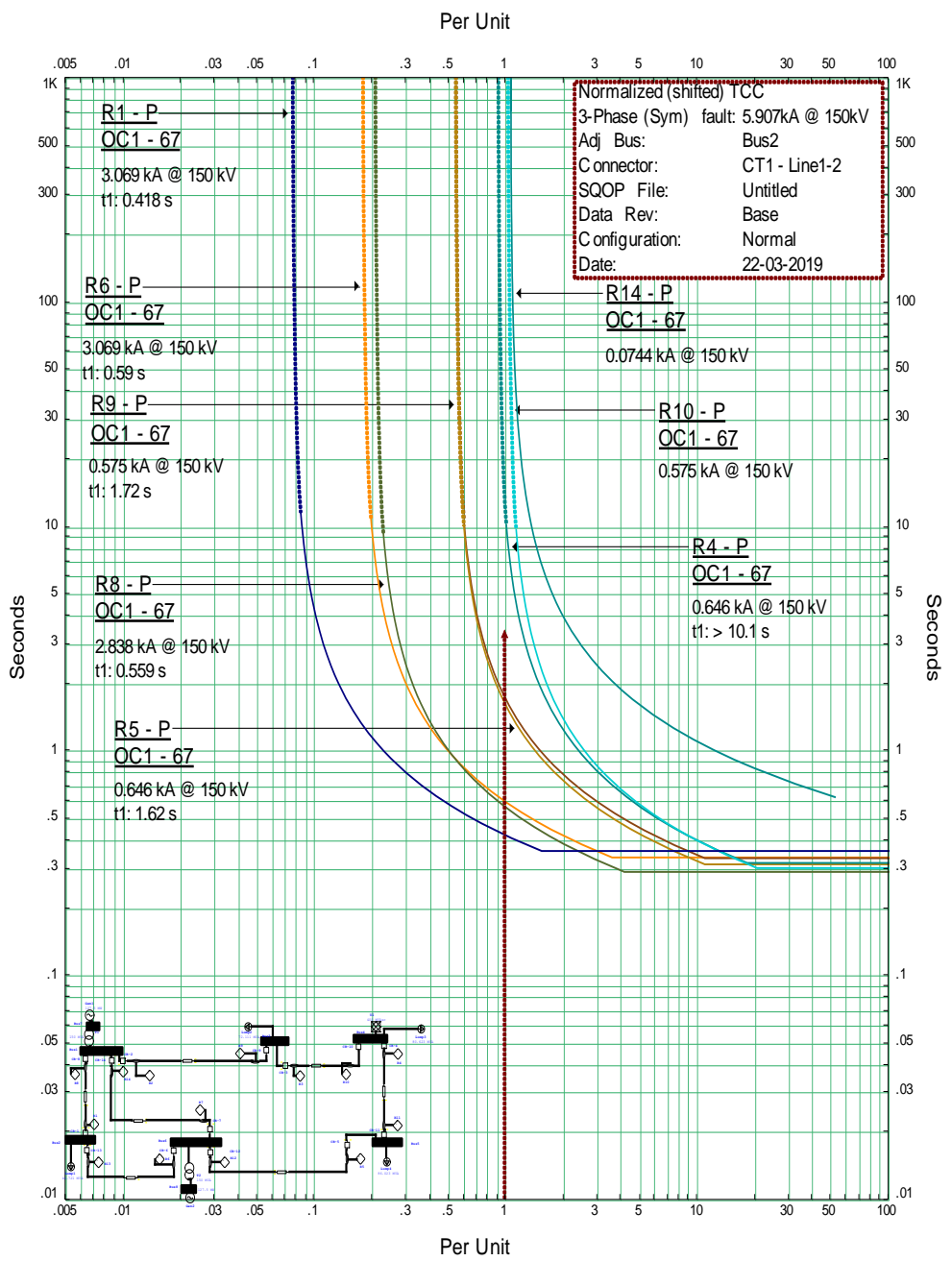

Figure 10. The fault at the transmission line (1-2) 


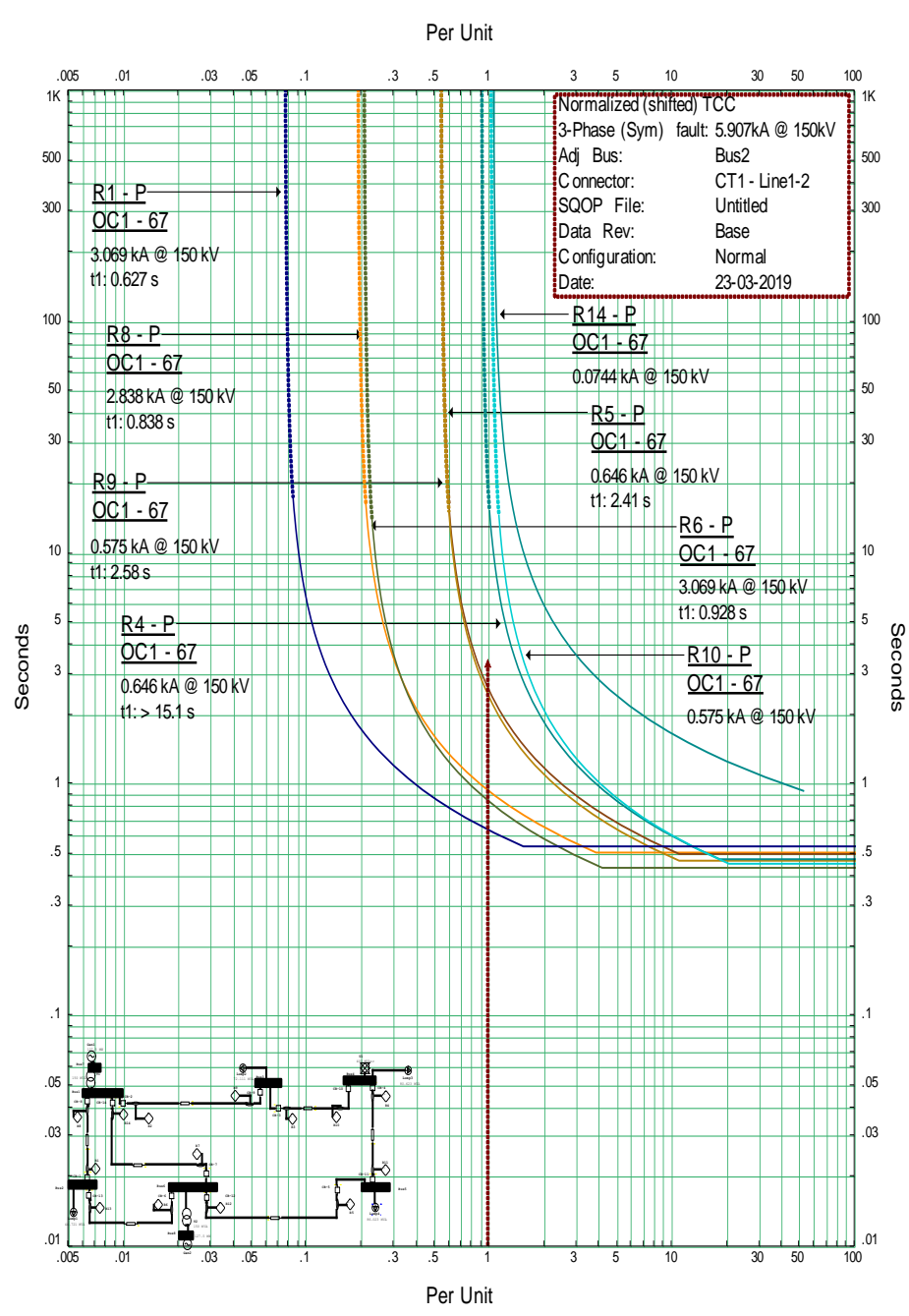

Figure 11. The fault at the transmission line (1-2) close-in of relay 1 (R1) (case 1) close-in of relay 1 (R1) (case 2)

\section{CONCLUSION}

In this paper, the operating time for the second zone of each distance relay set as independent value and the main aim was to obtain suitable coordination. Also, ETAP program has been used to obtain operating time for all DOC relays to validate it with the time which obtained from MATLAB simulation all operating times was identical and accurate in two cases 1 and 2.

So the independent setting for second zone operating time to each distance protection relay in the power systems is a better setting than a constant setting for all relays to ensure suitably coordination between DOC and distance relays. A nonlinear multivariable optimization technique was used with linear inequality constraints to obtain that with different performance indexes with and without pilot signal. So the active-set performance index in all cases with pilot signal was the best than other performance indexes to obtain optimal values at less time and less number of iterations.

\section{REFERENCES}

[1] C. Russell Mason, "The art and science of protective relaying", John Wiley \& Sons, 2015.

[2] Abdullah Hamed Ahmed, Ahmed Jasim Sultan, "A new approach of Mho distance relay for Transmission line protection" IOP Conference Series: Materials Science and Engineering, No.518, 2019.

[3] Salah K. El-Sayed, Hassan S. Mohamed, "Enhancing the performance of distance protection relays using interactive control system", Indonesian Journal of Electrical Engineering and Computer Science, Vol. 13, No. 1, January 2019. 
[4] S. Karupiah, M.H. Hussain, I. Musirin, S.R.A. Rahim, "Prediction of overcurrent relay miscoordination time using urtificial neural network", Indonesian Journal of Electrical Engineering and Computer Science, Vol. 14, No. 1, April 2019.

[5] Y. a. S. J. a. M. H. R. Damchi, "Optimal coordination of distance and directional overcurrent relays considering different network topologies," Iranian Journal of Electrical \& Electronic Engineering, vol. 11, pp. 231--240, 2015.

[6] Elmahdi Khoudry, Abdelaziz Belfqih, Tayeb Ouaderhman, Jamal Boukherouaa, Faissal Elmariami, "Multi-scale morphological gradient algorithm based ultra-high-speed directional transmission line protection for internal and external fault discrimination", International Journal of Electrical and Computer Engineering, Vol. 9, No. 5, October 2019

[7] Dazahra, M. N.; Elmariami, F.; Belfqih, A.; Boukherouaa, J, "Smart Local Backup Protection for Smart Substation", International Journal of Electrical and Computer Engineering, Vol. 7,No. 5, pp.2321-2328, October 2019.

[8] H. A. Abyaneh, S. S. H. Kamangar, F. Razavi and R. M. Chabanloo, "A new genetic algorithm method for optimal coordination of overcurrent relays in a mixed protection scheme with distance relays," 2008 43rd International Universities Power Engineering Conference, Padova, 2008, pp.1-5.

[9] Y. a. S. J. a. R. M. H. Damchi, "Preprocessing of distance and directional overcurrent relays coordination problem considering changes in network topology," International Transactions on Electrical Energy Systems, vol. 26, no. 2, pp. 32--48, 2016.

[10] M. Khederzadeh, "Back-up protection of distance relay second zone by directional overcurrent relays with combined curves," 2006 IEEE Power Engineering Society General Meeting, Montreal, Que., 2006, pp. 6 pp.-

[11] Y. a. S. J. a. M. H. R. Damchi, "Considering pilot protection in the optimal coordination of distance and directional overcurrent relays," Iran. J. Electr. Electron. Eng, vol. 11, pp. 154--164, 2015.

[12] S. a. N. F. a. S. N. a. B. M. Samadinasab, "Optimal Coordination of Overcurrent and Distance Relays Using Hybrid Differential Evolutionary and Genetic algorithms (DE-GA)," International Electrical Engineering Journal (IEEJ), vol. 6, pp. 1999-2008, 2015.

[13] J. Sadeh, V. Amintojjar and M. Bashir, "Coordination of overcurrent and distance relays using hybrid Particle Swarm Optimization," 2011 International Conference on Advanced Power System Automation and Protection, Beijing, 2011, pp. 1130-1134.

[14] S. S. H. a. A. H. A. a. R. F. a. C. R. M. Kamangar, "Optimal combined overcurrent and distance relays coordination using a new genetic algorithm method," Int. J. Innov. Energy Syst. Power, vol. 5, pp. 17--21, 2010.

[15] P. A. Bangar and A. A. Kalage, "Optimum coordination of overcurrent and distance relays using JAYA optimization algorithm," 2017 International Conference on Nascent Technologies in Engineering (ICNTE), Navi Mumbai, 2017, pp. 1-5.

[16] Ali Abbasi, Hossein Kazemi Karegar, Tohid Soleymani Aghdam, "Inter-trip links incorporate optimal protection coordination," International Journal of Electrical and Computer Engineering, Vol. 10. No. 1, pp. 72-79, Feberury 2020.

[17] S. Jamali and M. Pourtandorost, "New approach to coordination of distance relay zone-2 with overcurrent protection using linear programming methods," 39th International Universities Power Engineering Conference, 2004. UPEC 2004., Bristol, UK, 2004, pp. 827-831 vol. 1.

[18] M. a. K. F. a. K. A. Ghanbarian, "Setting Directional Over Current Relays as the Local Backup of Distance Relays in Power Network," in Fifteenth National Power Systems Conference (NPSC), 2008.

[19] G. Kobet et al., "Justifying pilot protection on transmission lines," 2010 63rd Annual Conference for Protective Relay Engineers, College Station, TX, 2010, pp. 1-31

[20] A. K. Pandey and S. Kirmani, "Implementation of genetic algorithm to find the optimal timing of overcurrent relays," 2016 IEEE International Power Electronics and Motion Control Conference (PEMC), Varna, 2016, pp. 400405.

[21] M.-T. Yang and J.-C. Gu, "Optimal coordination of automatic line switches for distribution systems," Energies, vol. 5, no. 4, pp. 1150-1174, 2012.

[22] G. Darji, A. Patel and R. P. Mehta, "Optimal Coordination of Directional Overcurrent Relays Using AI Algorithms and Comparison," in ICRISET2017. International Conference on Research and Innovations in Science, Engineering and Technology. Selected Papers in Engineering, EasyChair, 2017, pp. 81-89.

[23] M. F. Kotb, M. El-Saadawi and E. H. El-Desouky, "Protection Coordination Optimization for FREEDM (Future Renewable Electric Energy Delivery and Management) System," Journal of Electrical Engineering, vol. 6, pp. 161176, 2018.

[24] P. Sookrod and P. Wirasanti, "Overcurrent relay coordination tool for radial distribution systems with distributed generation," in 2018 5th International Conference on Electrical and Electronic Engineering (ICEEE), IEEE, 2018, pp. 13-17.

[25] A. a. G. S. a. N. D. Yazdaninejadi, S. Teimourzadeh and F. Aminifar, "Dual-setting directional overcurrent relays for protecting automated distribution networks," IEEE Transactions on Industrial Informatics, vol. 15, no. 2, pp. 730740, 2018. 